1

2

3

4 Li-li $\mathrm{Li}^{1,2}$, Jing-lin Wang ${ }^{3}$, Xiao-hua $\mathrm{Ma}^{1,2,4}$, Jin-song $\mathrm{Li}^{1,2}$, Xiao-fei Yang ${ }^{5}$, Wei-feng Shi ${ }^{6}$, Zhao-jun 5 Duan $^{1,2, \&}$

6

$7 \quad 1$ Key Laboratory for Medical Virology and Viral Diseases, National Health Commission of the 8 People's Republic of China, Beijing, China.

92 National Institute for Viral Disease Control and Prevention, China CDC, Beijing, China.

\section{A novel SARS-CoV-2 related virus with complex recombination isolated from bats in Yunnan province, China}
3. Yunnan Tropical and Subtropical Animal Viral Disease Laboratory, Yunnan Animal Science and Veterinary Institute, Kunming, Yunnan province, China;

4. School of Public Health, Gansu University of Chinese Medicine, Lanzhou, China

5. National Engineering Research Center of Freshwater Fisheries, Beijing Fisheries Research Institute

6. Key Laboratory of Etiology and Epidemiology of Emerging Infectious Diseases in Universities of Shandong, Shandong First Medical University, and Shandong Academy of Medical Sciences, Taian, China

\section{\&-Corresponding Author}

Corresponding author: Dr. Zhao-jun Duan. E-mail: zhaojund@126.com

Key words: SARS-CoV-2 related virus; Bats; CoVID-19; Recombination; Evolution 


\section{Abstract}

A novel beta-coronavirus, SARS-CoV-2, emerged in late 2019 and rapidly spread throughout the world, causing the COVID-19 pandemic. However, the origin and direct viral ancestors of SARS-CoV-2 remain elusive. Here, we discovered a new SARS-CoV-2-related virus in Yunnan province, in 2018, provisionally named $\operatorname{PrC31}$, which shares $90.7 \%$ and $92.0 \%$ nucleotide identities with SARS-CoV-2 and the bat SARSr-CoV ZC45, respectively. Sequence alignment revealed that several genomic regions shared strong identity with SARS-CoV-2, phylogenetic analysis supported that PrC31 shares a common ancestor with SARS-CoV-2. The receptor binding domain of PrC31 showed only $64.2 \%$ amino acid identity with SARS-CoV-2. Recombination analysis revealed that $\operatorname{PrC31}$ underwent multiple complex recombination events within the SARS-CoV and SARS-CoV-2 sub-lineages, indicating the evolution of PrC31 from yet-to-be-identified intermediate recombination strains. Combination with previous studies revealed that the beta-CoVs may possess more complicated recombination mechanism. The discovery of PrC31 supports that bats are the natural hosts of SARS-CoV-2.

\section{.}

.

.

5

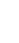

.

8


Introduction

mammalian and bird species $(1,2)$. So far, seven CoV species have been identified in humans. Of

these, severe acute respiratory syndrome coronavirus (SARS-CoV) emerged in 2003 and caused

multiple epidemics worldwide, and had a fatality rate of $\sim 9.5 \%(3)$. Approximately ten years later,

December 2019, a novel beta-CoV, now termed severe acute respiratory syndrome coronavirus 2

(SARS-CoV-2), was first identified. SARS-CoV-2 caused a pneumonia outbreak in Wuhan, China,

and eventually caused a pandemic, with $>116,521,000$ reported cases and $>2,589,000$ deaths

worldwide as of March 9, 2021 (7-10).

(11, 14-16), suggesting that bats may be the natural hosts of SARS-CoV. Similarly, several 
stored frozen samples $(7,10,14,19-22)$. Of these, RaTG13 and RmYN02, which were identified in

with SARS-CoV-2, respectively $(7,19)$. SARS-CoV-2-related CoVs were also identified in pangolins,

whose receptor binding domain (RBD) shared up to $97.4 \%$ nucleotide identity with that of

SARS-CoV-2 $(20,21)$. This suggests that pangolins are a potential host of SARS-CoV-2, although

the role of pangolins in the evolutionary history of SARS-CoV-2 remains elusive. Nevertheless,

either the direct progenitor of SARS-CoV-2 is yet to be discovered, or the transmission route of

SARS-CoV from bats to humans via an intermediate host must still be determined (24). The

discovery of more SARS-CoV-2-related viruses will help to clarify the details regarding the

emergence and evolutionary history of SARS-CoV-2.

Results

Identification of a novel SARS-CoV-2-related coronavirus

Based on the molecular identification results, all collected bats belonged to five different

species: Rhinolophus affinis, Miniopterus schreibersii, Rhinolophus blythi, Rhinolophus pusillus,

and Hipposideros armiger. By retrospectively analyzing our NGS data, we found a new bat

beta-CoV related to SARS-CoV-2 in Rhinolophus blythi collected from Yunnan province, China, in

2018. The qRT-PCR results revealed that two samples tested positive for SARS-CoV-2 with $C t$ 
values of 32.4 (sample C25) and 35.6 (sample C31). Both bats were identified as Rhinolophus blythi. A near complete genome of this virus comprising $29,749 \mathrm{bp}$ was obtained from sample C31 and tentatively named PrC31. The virus genome isolated from the second positive sample had the same sequence as $\operatorname{PrC31.}$

\section{Genetic characteristics and comparison with SARS-CoV-2 and other related viruses}

Analysis of the complete PrC31 genome revealed that it shared $90.7 \%$ and $92.0 \%$ nucleotide identity to SARS-CoV-2 and bat SARSr-CoV ZC45, respectively (Table 1). Although the whole genome of $\operatorname{PrC} 31$ was more closely related to ZC45 compared to the other viruses examined, several genes of $\operatorname{PrC31}$ showed highly similar nucleotide identities (> 96\%) with SARS-CoV-2, including E, ORF7a, ORF7b, ORF8, N and ORF10 (Table 1). Notably, ORF8 and ORF1a (the region spanning nucleotides 1-12719) of PrC31 were genetically closer to SARS-CoV-2 than any other viruses identified to date, exhibiting $98.1 \%$ and $96.6 \%$ nucleotide identities, respectively. However, in other regions, PrC31 was more similar to SARS-CoV or SARSr-CoV ZC45.

\footnotetext{
acid identity, whereas it was almost identical to that of $\mathrm{ZC45}$, with only one amino acid difference.
}

Similar to most bat SARSr-CoVs, one long ( 14 aa) deletion and one short ( 5 aa) deletion were present in PrC31, which were absent from SARS-CoV, SARS-CoV-2, pangonlin-CoV and RaTG13. We predicted the three-dimensional structure of the RBD of PrC31, ZC45 and SARS-CoV-2 using homology modeling. Similar to RmYN02, the two loops close to the receptor binding site of the 
112 PrC31 RBD were shorter than those of SARS-CoV-2, due to two deletions; this region may

113 influence the binding capacity of the PrC31 RBD with the angiotensin converting enzyme 2 (ACE2)

114 receptor (Fig.1A-1D). Moreover, of the six amino acid residues that are essential for the binding

115 of the SARS-CoV-2 spike protein to ACE2 (L455, F486, Q493, S494, N501, and Y505), PrC31 and

116 RmYN02 possesed only one( Y505) (Fig.1E)

117

Phylogenetic analysis of PrC31 and representative sarbecoviruses

Phylogenetic analysis of the complete PrC31 genome revealed that it belonged to a

separate clade to SARS-CoV-2, while most other SARS-CoV-2-related viruses were grouped

together (Fig.2). However, the PrC31 RNA-dependent RNA polymerase was phylogenetically

grouped within the SARS-CoV lineage and clustered with bat SARS-rCoV. The spike protein of

PrC31 fell within the SARS-CoV-2 sub-lineage and clustered with ZC45 and CXZ21, while being

distant from SARS-CoV-2. The topological differences between various regions of PrC31 strongly

suggest the occurrence of recombination events throughout its evolution.

Multiple and complex recombination events in the evolution of PrC31

Figure 3, three recombination breakpoints were detected. For the region spanning nucleotides 
133

$1-12,719$ and 27,143 to the $3^{\prime}$ terminus of the genome, PrC31 was most closely related to SARS-CoV-2 and RmYN02. In these regions, PrC31 was phylogenetically grouped with RmYN02 and in a sister clade to SARS-CoV-2 (Figure 4a and 4d). For the 12,720-20,244 nucleotide region, which included ORF1ab, PrC31 was grouped with SARS-CoV and bat SARSr-CoVs (Figure 4b). Moreover, PrC31 presented the highest similarity to ZC45 in the $20,245-27,142$ genomic fragment, which included part of ORF1ab, S, ORF3, E, and part of the M gene, and fell within the SARS-CoV-2 sub-lineage (Figure 4c)

\section{Discussion}

The recently-emerged SARS-CoV-2 virus triggered the ongoing COVID-19 pandemic, which has high morbidity and fatality rates, and poses a great threat to global public health. Identifying the origin and host range of SARS-CoV- 2 will aid in its prevention and control, and will facilitate preparation for future CoV pandemics. Although several SARS-CoV-2-related viruses were detected in bats and pangolins, none of them appear to be the immediate ancestor of SARS-CoV-2; the exact origin of SARS-CoV-2 is still unclear $(12,25)$. In this study, we discovered PrC31, a sarbecovirus isolated from bat intestinal tissues collected in 2018. PrC31 phylogenetically falls into the SARS-CoV-2 clade and has undergone multiple and complex recombination events.

Animals that continuously harbor viruses closely related to SARS-CoV-2 for extended time periods can become natural SARS-CoV-2 hosts (2). To date, several bat viruses have been 
identified that have strong sequence similarities to SARS-CoV-2, sharing more than $90 \%$ sequence identity. Especially, RaTG13 possesses $96.2 \%$ identity with SARS-CoV-2 $(7,18,19,21,23)$. The PrC31 virus identified in this study showed $90.7 \%$ genome identity with SARS-CoV-2; notably, the E, ORF7, ORF8, N and ORF10 genes shared more than $96 \%$ identity with SARS-CoV-2. Both the genetic similarity and diversity of SARS-CoV-2-related viruses support the claim that bats were the natural hosts of SARS-CoV-2 $(10,19)$.

Recombination events between various SARSr-CoVs have occurred frequently in bats $(5,16)$. SARS-CoV-2 may also be a recombined virus, potentially with the backbone of RaTG13 and a RBD region acquired from pangolin-like $\operatorname{SARSr-CoVs}(12,21)$. In this study, we found that PrC31 phylogenetic clustered with SARS-CoV-2 and its related viruses. The results from our phylogenetic analyses suggested that recombination had occurred in PrC31. The similarity plot indicated that the PrC31 was subjected to multiple and complex recombination events involving more than two sarbecoviruses in the SARS-CoV and SARS-CoV-2 sub-lineages. The three breakpoints of PrC31 separate the genome into four regions. Region 1 (within ORF1a) and region 4 of $\operatorname{PrC} 31$ were closely related to SARS-CoV-2, RaTG13 and RmYN02. Region 2 of PrC31 was more similar to members of the SARS-CoV sub-lineage, including SARS-CoV and SARSr-CoV Rs4237 strain; region 3 was more closely related to ZC45 within SARS-CoV-2 sub-lineage. The multiple recombination events of PrC31 hint toward the existence of intermediate recombination strains within the SARS-CoV and SARS-CoV-2 sub-lineages that are yet to be identified. Our work suggests that the backbone of PrC31 may have evolved from a recent common ancestor of RaTG13, RmYN02 and 
175

176

SARS-CoV-2, and that it acquired regions 2 and 3 from precursor viruses of SARS-CoV and SARSr-CoV ZC45, respectively.

At present, the precise patterns and mechanisms driving recombination in sarbecoviruses are largely unknown. A recent report identified 16 recombination breakpoints in 69 sarbecoviruses (26), although in the majority of strains, the recombination sites were located within the $S$ gene and upstream of $\operatorname{ORF8}(5,9,16)$. The three recombination breakpoints of $\operatorname{PrC31}$ were located in ORF1a, ORF1b and M genes with long fragment recombination, suggestive of a complicated recombination pattern in sarbecoviruses. Similar to PrC31, SARS-CoV-2 may have evolved via complex recombination between various related coronaviruses or their progenitors (10). In fact, the direct progenitor of SARS-CoV may have evolved by recombination with progenitors of SARSr-CoV (Hu et al. 2017). Together, these findings suggest that recombination and its role in the evolution history of sarbecoviruses may be more complicated and significant than initially expected.

Pangolins may also harbor ancestral beta-CoVs related to SARS-CoV-2 $(2,20,21)$; the receptor-binding motif of pangolin beta-CoVs share an almost identical amino acid sequence with SARS-CoV-2 $(20,21)$, suggesting that SARS-CoV-2 may have acquired its RBD region from a pangolin CoV via recombination(27). However, unlike bats, pangolins infected with beta-CoVs present overt symptoms and eventually die, rendering them unlikely to be natural hosts. Intermediate hosts generally serve as zoonotic sources for human infection, acting as vectors for viral replication and transmission to humans (2). Current evidence suggests that pangolins were not the direct intermediate hosts of SARS-CoV-2. However, pangolins certainly played an 
to the virus presumed as "SARS-CoV-3", which may be transmitted to human populations in the future.

of SARS-CoV-2. It will be necessary to expand the sampling areas and animal species examined to

find more close relatives of SARS-CoV-2. There may be an unknown intermediate host of

kit, which targets the ORF1ab and N genes of SARS-CoV-2. This emphasizes the need to gather

samples may be contaminated with a closely related beta-CoVs from wild animals such as bats.

\section{Materials and methods}




\section{Sample collection and pretreatment}

218 In 2018, 36 bats were captured in Yunnan province, China. The bats were dissected following anesthetization. Liver, lung, spleen and intestinal tissue specimens were collected and transported to the Chinese center for disease control, where they were stored at $-80^{\circ} \mathrm{C}$ until further analysis. The bat species were identified by polymerase chain reaction (PCR) to amplify the cytochrome B gene, as previously described (29). Intestinal tissues collected from 36 bats were homogenized in minimum essential medium and the suspensions were centrifuged at 8,000 rpm. The supernatants were merged into two pools according to bat species, then digested using DNase I for RNA Extraction. All procedures were performed in a biosafety cabinet in a biosafety level 2 facility. This study was approved by the ethics committee of the CCDC, and was performed according to Chinese ethics, laws and regulations. construct the sequencing libraries. The library preparation and sequencing steps were performed by Novogene Bioinformatics Technology (Beijing, China). In brief, the ribosomal RNA was removed using the Ribo-Zero-Gold (Human-Mouse-Rat) Kit (Illumina, USA) and the 


\section{Bioinformatic analyses}

Bioinformatics analysis of the sequencing data was conducted using an in-lab bioinformatics analysis platform. Prinseq-lite software (version 0.20 .4 ) was used to remove lower quality reads, and Bowtie2 was used to align and map the filtered reads to the host reference genome. Mira (version 4.0.2) was used for de novo assembly of the clean reads. Both BLASTn and BLASTx of the BLAST+ package (version 2.2.30) were used to search against local viral nucleotide and protein databases. The E-value cut-off was set to $1 \times 10^{-5}$ to maintain high sensitivity and a low false-positive rate when performing BLAST searches.

\section{Sequencing of full-length genomes and quantitative real-time PCR (qRT-PCR)}

We obtained reads that showed $96-98 \%$ nucleotide identity to SARS-CoV-2 from the PrC31 genome library. To confirm the sequences obtained from NGS and to fill the gaps, we designed 32 primer pairs according to the consensus sequences from the NGS and the conserved regions of SARS-CoV-2, RaTG13 and RmYN02, to amplify the whole PrC31 genome with at least $100 \mathrm{bp}$ overlap between adjacent PCR fragments (Table S1). The PCR products were subjected to Sanger sequencing with pair-end sequencing. The $25 \mathrm{bp}$ at the $5^{\prime}$ and $3^{\prime}$ termini were omitted, and the remaining sequences were assembled using Geneious Prime. Positive samples were quantified using TaqMan-based qPCR kit targeting the ORF1ab and N genes (BioGerm, China).

\section{Phylogenetic and recombination analyses}

The complete genome sequences of reference viruses were downloaded from GenBank 
259 (https://www.ncbi.nlm.nih.gov/) and GISAID (https://www.gisaid.org/). The complete genome of

260 PrC31 was aligned with representative SARS-CoV, SARS-CoV-2 and SARSr-CoV using Mafft

261 (v7.475). Phylogenetic analyses were performed with RaxML software (v8.2.11) using the general

262 time reversible nucleotide substitution model, GAMMA distribution of rates among sites, and

2631000 bootstrap replicates. Potential recombination events were screened using RDP4 software

264 and further analyzed by similarity plot using Simplot (v3.5.1) with potential major and minor 265 parents.

\section{Structural modeling}

The three-dimensional structures of PrC31, ZC45 and SARS-CoV-2 RBDs were modeled with the

Swiss-Model program using the SARS-CoV-2 RBD structure (PDB: 7a91.1) as the template.

Data availability

272 The sequences of PrC31 generated in this study were deposited in the GISAID and GenBank

databases with the accession numbers EPI_ISL_1098866 and MW703458, respectively.

Acknowledgements

This study was supported by National Science and Technology Major Project of China

(No.2018ZX10305409-004-002)

\section{Author contributions}

278 L-L L, Acquisition of data, Analysis and interpretation of data, Conception and design, Drafting or

279 revising the article; $\mathrm{M}-\mathrm{XH}$, Acquisition of data; J-S L, Conception and design experiment; J-L W,

280 Sample collection, Acquisition of data. W-F S, Analysis and interpretation of data, Conception and

281 design, Drafting or revising the article. Z-J D, Conception and design, Analysis and interpretation 
282 of data, Drafting or revising the article.

283

284

285 Table 1: Sequence identities comparing PrC31 with SARS-CoV-2 and other representative

286 beta-CoVs

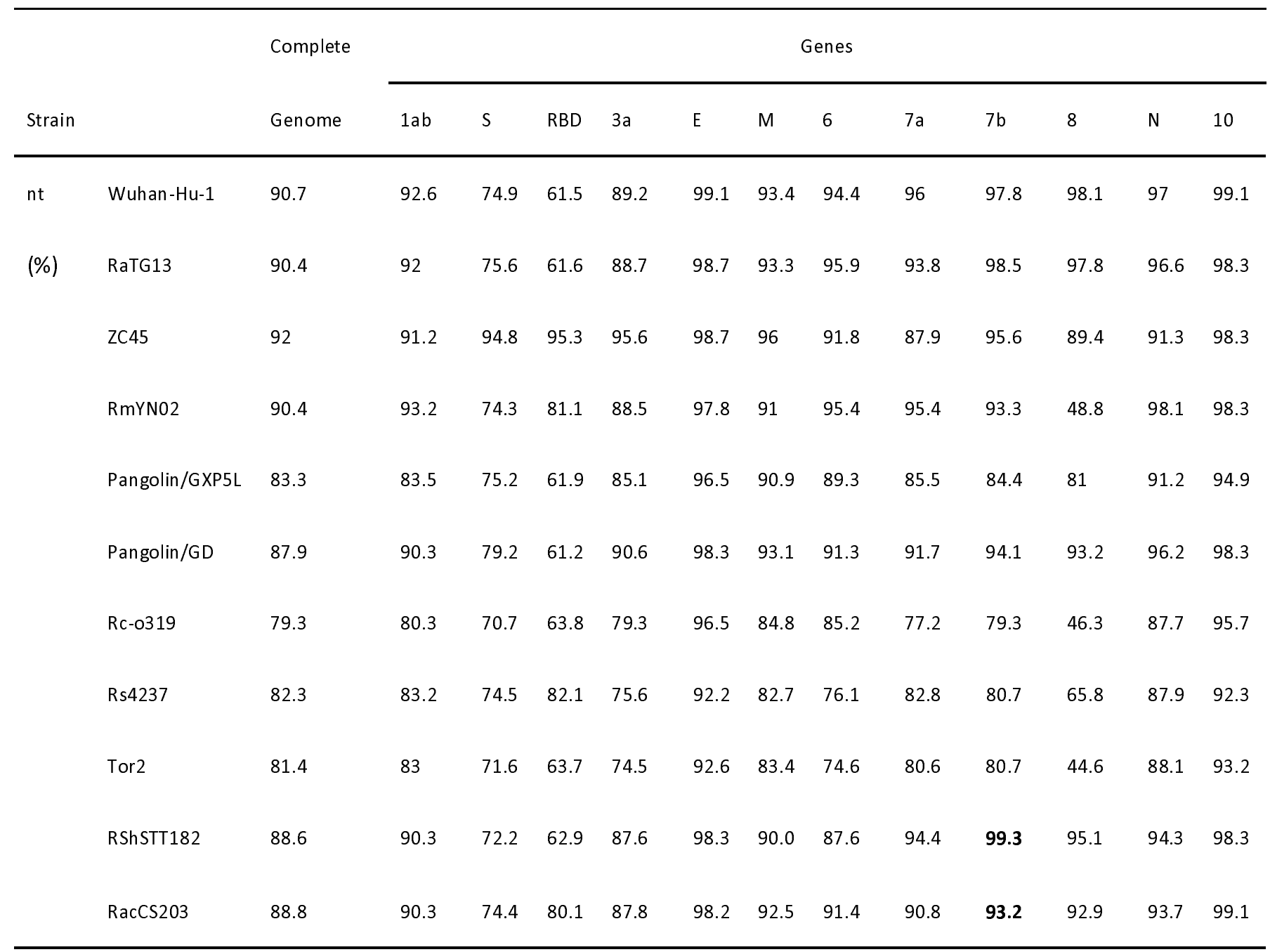


Figure legends

Figure 1: Homology modeling structures and Characterization of Receptor binding domain (RBD)

of PrC31 and Representative Beta-CoVs. (A-D) Homology modeling structures of PrC31 and

Representative Beta-CoVs. The three-dimensional structures of PrC31, ZC45 and SARS-CoV-2

RBDs were modeled using the Swiss-Model program, using the SARS-CoV-2 RBD structure (PDB:

7a91.1) as a template. The two deletion loops in PrC31 and ZC45 are marked with a circle. (E)

Characterization of the RBDs of PrC31 and representative beta-CoVs. The six critial amino acid

residues for ACE2 interaction were marked using red star.

302

Fig. 2 Phylogenetic trees of SARS-CoV-2 and representative sarbecoviruses. (A)Complete genome;

(B)RdRp gene (C)S gene (D)RBD region. SARS-CoV lineage and SARS-CoV-2-related lineages are

shown in orange and purple shadow, respectively. Viruses that originated in bats are labeled in

blue, human viruses are labeled in red and pangolin viruses are labeled in green. The PrC31

identified in this study is highlighted in yellow shadow. Phylogenetic analyses were performed

with RaxML software (v8.2.11) using the GTR nucleotide substitution model, GAMMA distribution

of rates among sites, and 1000 bootstrap replicates 
313 PrC31as the query. Slide window was set to $1000 \mathrm{bp}$ with $100 \mathrm{bp}$ steps.

314

Fig. 4 Phylogenetic trees of various regions of the PrC31 genome. SARS-CoV and SARS-CoV-2-related lineages are shown as orange and purple shadow, respectively. The PrC31 virus identified in this study is indicated with yellow shadow. Viral taxonomy is labeled in color that originated in bats are labeled in blue, humans in red, and pangolins in green. Phylogenetic analyses were performed with RaxML software (v8.2.11) using the GTR nucleotide substitution model, GAMMA distribution of rates among sites, and 1000 bootstrap replicates

\section{References}

1. Corman VM, Muth D, Niemeyer D, Drosten C. Hosts and Sources of Endemic Human Coronaviruses. Adv Virus Res. 2018;100:163-88. doi:10.1016/bs.aivir.2018.01.001

2. Ye ZW, Yuan S, Yuen KS, Fung SY, Chan CP, Jin DY. Zoonotic origins of human coronaviruses. Int J Biol Sci. 2020;16(10):1686-97. doi:10.7150/ijbs.45472

3. CDC. Severe Acute Respiratory Syndrome: Available online:https://www.cdc.gov/sars/about/fs-sars.html;

4. Banerjee A, Kulcsar K, Misra V, Frieman M, Mossman K. Bats and Coronaviruses. Viruses. 2019;11(1). doi:10.3390/v11010041

5. Cui J, Li F, Shi ZL. Origin and evolution of pathogenic coronaviruses. Nat Rev Microbiol. 2019;17(3):181-92. doi:10.1038/s41579-018-0118-9

6. Woo PC, Lau SK, Huang Y, Yuen KY. Coronavirus diversity, phylogeny and interspecies jumping. Exp Biol Med (Maywood). 2009;234(10):1117-27. doi:10.3181/0903-MR-94

7. Zhou P, Yang XL, Wang XG, Hu B, Zhang L, Zhang W, et al. A pneumonia outbreak associated 
338 with a new coronavirus of probable bat origin. Nature. 2020;579(7798):270-3.

339 doi:10.1038/s41586-020-2012-7

340 8. Zhu N, Zhang D, Wang W, Li X, Yang B, Song J, et al. A Novel Coronavirus from Patients with

341 Pneumonia in China, 2019. N Engl J Med. 2020;382(8):727-33. doi:10.1056/NEJMoa2001017

342 9. Wu F, Zhao S, Yu B, Chen YM, Wang W, Song ZG, et al. A new coronavirus associated with 343 human respiratory disease in China. Nature. 2020;579(7798):265-9. doi:10.1038/s41586-020-2008-3

344 10. Hu B, Guo H, Zhou P, Shi ZL. Characteristics of SARS-CoV-2 and COVID-19. Nat Rev Microbiol. 345 2020. doi:10.1038/s41579-020-00459-7

346 11. Lau SK WP, Li KS, Huang Y, Tsoi HW, Wong BH, Wong SS, Leung SY, Chan KH, Yuen KY. Severe 347 acute respiratory syndrome coronavirus-like virus in Chinese horseshoe bats. Proc Natl Acad Sci U S A. $348 \quad 2005 ; 102 \quad 14040-5$

349 12. Lau SKP, Luk HKH, Wong ACP, Li KSM, Zhu L, He Z, et al. Possible Bat Origin of Severe Acute

350 Respiratory Syndrome Coronavirus 2. Emerg Infect Dis. 2020;26(7):1542-7.

351 doi:10.3201/eid2607.200092

352 13. Menachery VD, Yount BL, Jr., Debbink K, Agnihothram S, Gralinski LE, Plante JA, et al. A 353 SARS-like cluster of circulating bat coronaviruses shows potential for human emergence. Nat Med. 354 2015;21(12):1508-13. doi:10.1038/nm.3985

355 14. Hu D, Zhu C, Ai L, He T, Wang Y, Ye F, et al. Genomic characterization and infectivity of a novel 356 SARS-like coronavirus in Chinese bats. Emerg Microbes Infect. 2018;7(1):154. 357 doi:10.1038/s41426-018-0155-5

358 15. Li W SZ, Yu M, Ren W, Smith C, Epstein JH, Wang H, Crameri G, Hu Z, Zhang H, Zhang J, 359 McEachern J, Field H, Daszak P, Eaton BT, Zhang S, Wang LF. Bats are natural reservoirs of SARS-like 360 coronaviruses. Science. 2005;310(5748):4

361 16. Hu B, Zeng LP, Yang XL, Ge XY, Zhang W, Li B, et al. Discovery of a rich gene pool of bat 362 SARS-related coronaviruses provides new insights into the origin of SARS coronavirus. PLoS Pathog. 363 2017;13(11):e1006698. doi:10.1371/journal.ppat.1006698

364 17. Forni D, Cagliani R, Clerici M, Sironi M. Molecular Evolution of Human Coronavirus Genomes. 365 Trends Microbiol. 2017;25(1):35-48. doi:10.1016/j.tim.2016.09.001 
366 18. Hul V, Delaune D, Karlsson EA, Hassanin A, Tey PO, Baidaliuk A, et al. A novel SARS-CoV-2

367 related coronavirus in bats from Cambodia. bioRxiv 2021. doi:10.1101/2021.01.26.428212

368 19. Zhou H, Chen X, Hu T, Li J, Song H, Liu Y, et al. A Novel Bat Coronavirus Closely Related to

369 SARS-CoV-2 Contains Natural Insertions at the S1/S2 Cleavage Site of the Spike Protein. Curr Biol.

$370 \quad$ 2020;30(11):2196-203 e3. doi:10.1016/j.cub.2020.05.023

371 20. Xiao K, Zhai J, Feng Y, Zhou N, Zhang X, Zou JJ, et al. Isolation of SARS-CoV-2-related coronavirus

372 from Malayan pangolins. Nature. 2020;583(7815):286-9. doi:10.1038/s41586-020-2313-x

373 21. Lam TT, Jia N, Zhang $\mathrm{YW}$, Shum MH, Jiang JF, Zhu HC, et al. Identifying SARS-CoV-2-related

374 coronaviruses in Malayan pangolins. Nature. 2020;583(7815):282-5. doi:10.1038/s41586-020-2169-0

375 22. Murakami S, Kitamura T, Suzuki J, Sato R, Aoi T, Fujii M, et al. Detection and Characterization of

376 Bat Sarbecovirus Phylogenetically Related to SARS-CoV-2, Japan. Emerg Infect Dis. 2020;26(12):3025-9.

377 doi:10.3201/eid2612.203386

378 23. Wacharapluesadee S TC, Maneeorn P, Duengkae P, Zhu F, Joyjinda Y, Kaewpom T, Chia WN,

379 Ampoot W, Lim BL, Worachotsueptrakun K, Chen VC, Sirichan N, Ruchisrisarod C, Rodpan A, 380 Noradechanon K, Phaichana T, Jantarat N, Thongnumchaima B, Tu C, Crameri G, Stokes MM, 381 Hemachudha T, Wang LF. . Evidence for SARS-CoV-2 related coronaviruses circulating in bats and pangolins in Southeast Asia. Nat Commun. 2021;12(1):972

383 24. Banerjee A, Doxey AC, Mossman K, Irving AT. Unraveling the Zoonotic Origin and Transmission 384 of SARS-CoV-2. Trends Ecol Evol. 2021;36(3):180-4. doi:10.1016/j.tree.2020.12.002

385 25. Andersen KG, Rambaut A, Lipkin WI, Holmes EC, Garry RF. The proximal origin of SARS-CoV-2. 386 Nature Medicine. 2020;26(4):450-2. doi:10.1038/s41591-020-0820-9

387 26. Spyros Lytras JH, Wei Xia, Xiaowei Jiang, David L Robertson. Exploring the natural origins of 388 SARS-CoV-2. bioRxiv. 2021;01(22):427830

389 27. Zhang T, Wu Q, Zhang Z. Probable Pangolin Origin of SARS-CoV-2 Associated with the COVID-19

390 Outbreak. Curr Biol. 2020;30(7):1346-51 e2. doi:10.1016/j.cub.2020.03.022

391 28. Zhang YZ, Holmes EC. A Genomic Perspective on the Origin and Emergence of SARS-CoV-2. Cell. 392 2020;181(2):223-7. doi:10.1016/j.cell.2020.03.035

393 29. Irwin DM KT, Wilson AC. Evolution of the cytochrome b gene of mammals. J Mol Evol. 
bioRxiv preprint doi: https://doi.org/10.1101/2021.03.17.435823; this version posted March 19, 2021. The copyright holder for this preprint (which was not certified by peer review) is the author/funder, who has granted bioRxiv a license to display the preprint in perpetuity. It is made available under aCC-BY-NC-ND 4.0 International license.

$394 \quad 1991 ; 32(2): 128-44$

395 
A

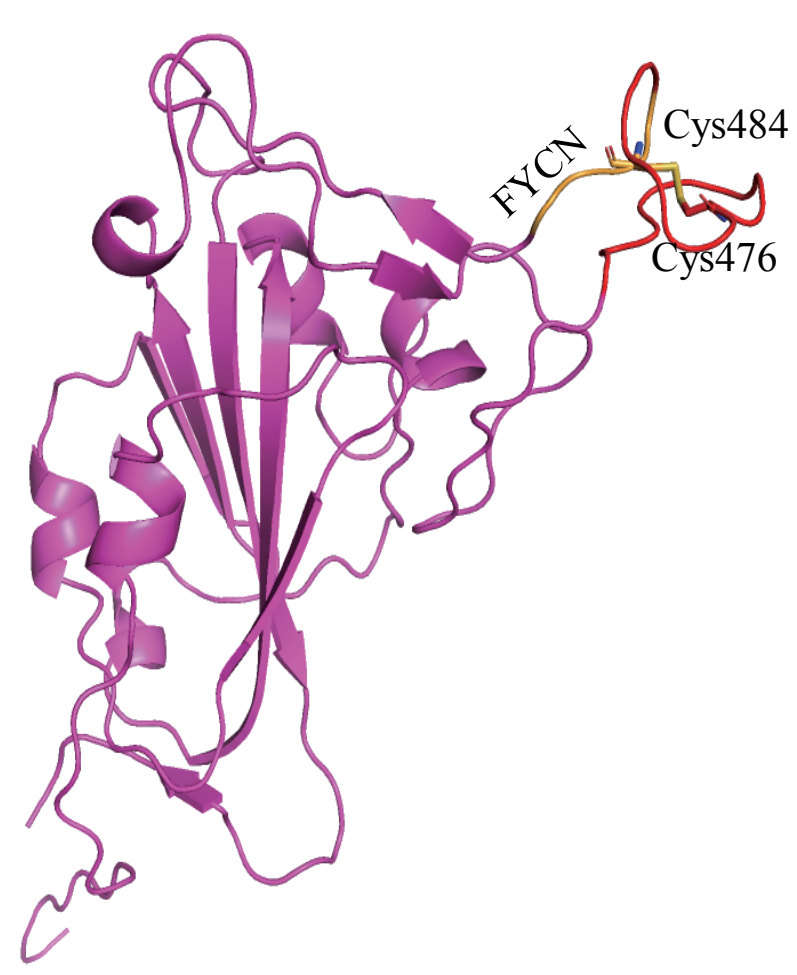

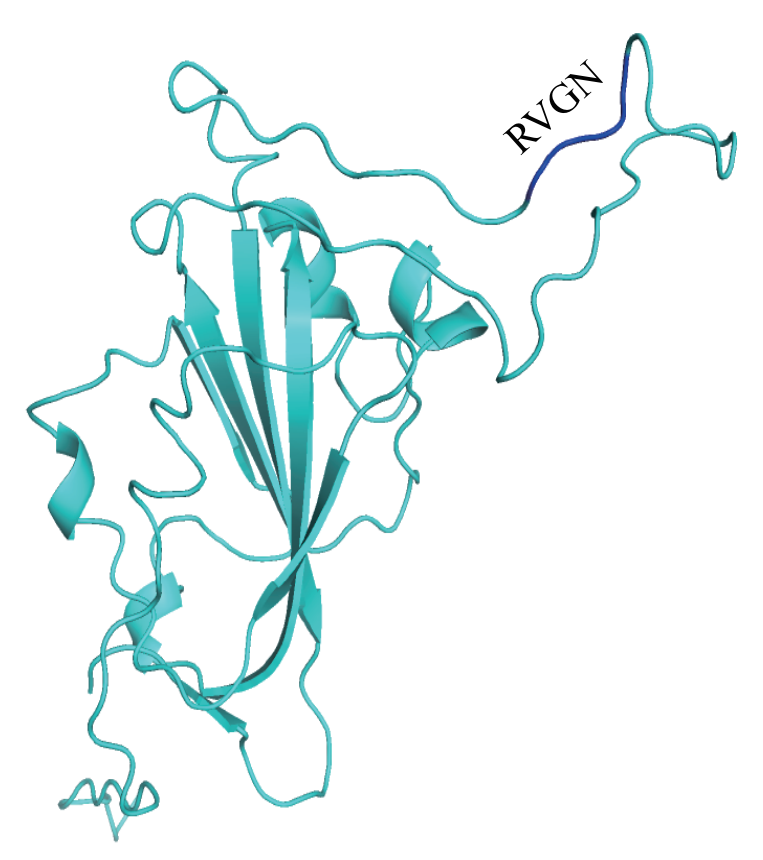

t
D SARS-CoV-2/PrC31/ZC45

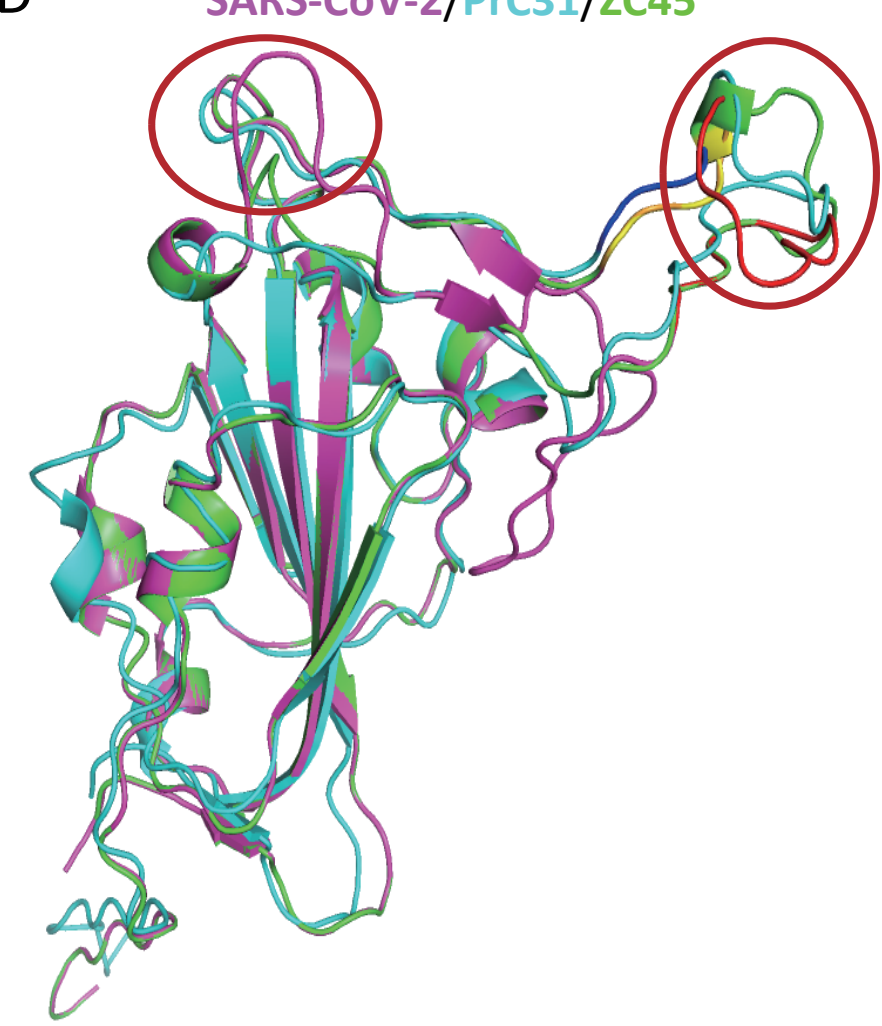

E

Wuhan-Hu-1 pangolin/GD Pangolin/GX RaTG13

Tor2

SARS-A001

RshSTT182

Rc-0319

PrC31

ZC45

Rs 4237

RmYN02
사산
NSNNLDSKVGGNYNYLYRLFRKSNLKPFERD ISTEI YQAGSTPCNGVEGENCYFPLQSYGFQPTNGVGYQP NSNNLDSKVGGNYNYLYRLFRKSNLKPFERD ISTEI YQAGSTPCNGVEGFNCYFPLQS YGFHPTNGVGYQP NSVKQDALTGGNYGYLYRLFRKSKLKPFERD ISTE I YQAGSTPCNGQVGLNCYYPLERYGFHPTTGVNYQP NSKH I DAKEGGN F N YLYRL FRKANLKPFERD ISTE I YQAGSKPCNGQTGLNCYYPLYRYGFYPTDGVGHQP NTRN I DATSTGNYN YKYR Y LRHGKLRP FERD I S NVPFSPDGKPCT - P P L L C YWPLNDYGFYT TTG I G YQP NTRN I DATSTGN YN YKHRYLRHGKLRP FERD I SNVPFSPEGKPCT - PPAPNCYWPLRGYGFYTTSG I GYQP NS ISLDAGG - - - S Y YYRLFRKSVLKPFERD ISTQLYQAGDKPCS - V EGPDCYYPLQSYYFQSTNGVGYQP NSRNQDASTS GNFNYYYR I WRSEKLRP FERD I AHYDYQVGT - . - . . - . QFKSSLKNYGFYSSAGDSHQP NTAKQDVG - . . - S Y F YRSHRSTKLKP FERDLSSDE . . . . . . . . . . . INGVRTLSTYDFNPNVPLEYQA NTAKQDVG - . - . NYFYRSHRSTKLKPFERDLSSDE - . . . . . . . . - - . NGVRTLSTYDFNPNVPLEYQA NTAKQDQG - . . - QYYYYRSRKTKLKPFERDLSSDE - . . . . . . . . . . NGVRTLSTYDFYPTVPIEYQA NTAQQDIG . . . . SYFYRSHRAVKLKPFERDLSSDE . . . . . . . . . . . NGVRTLSTYDFNPNVPLDYQA 


\section{A: Complete genome}

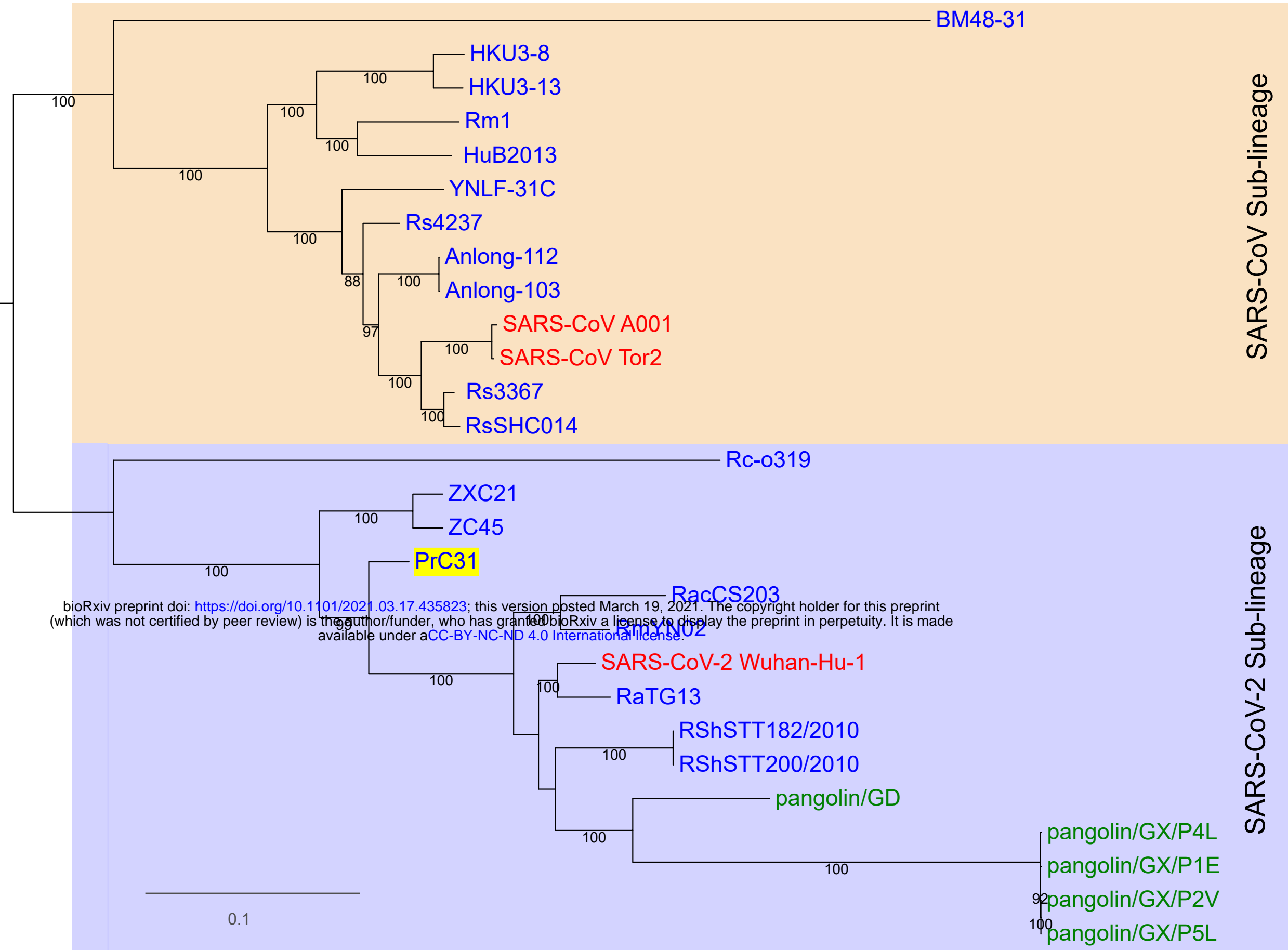

\section{C: S gene}

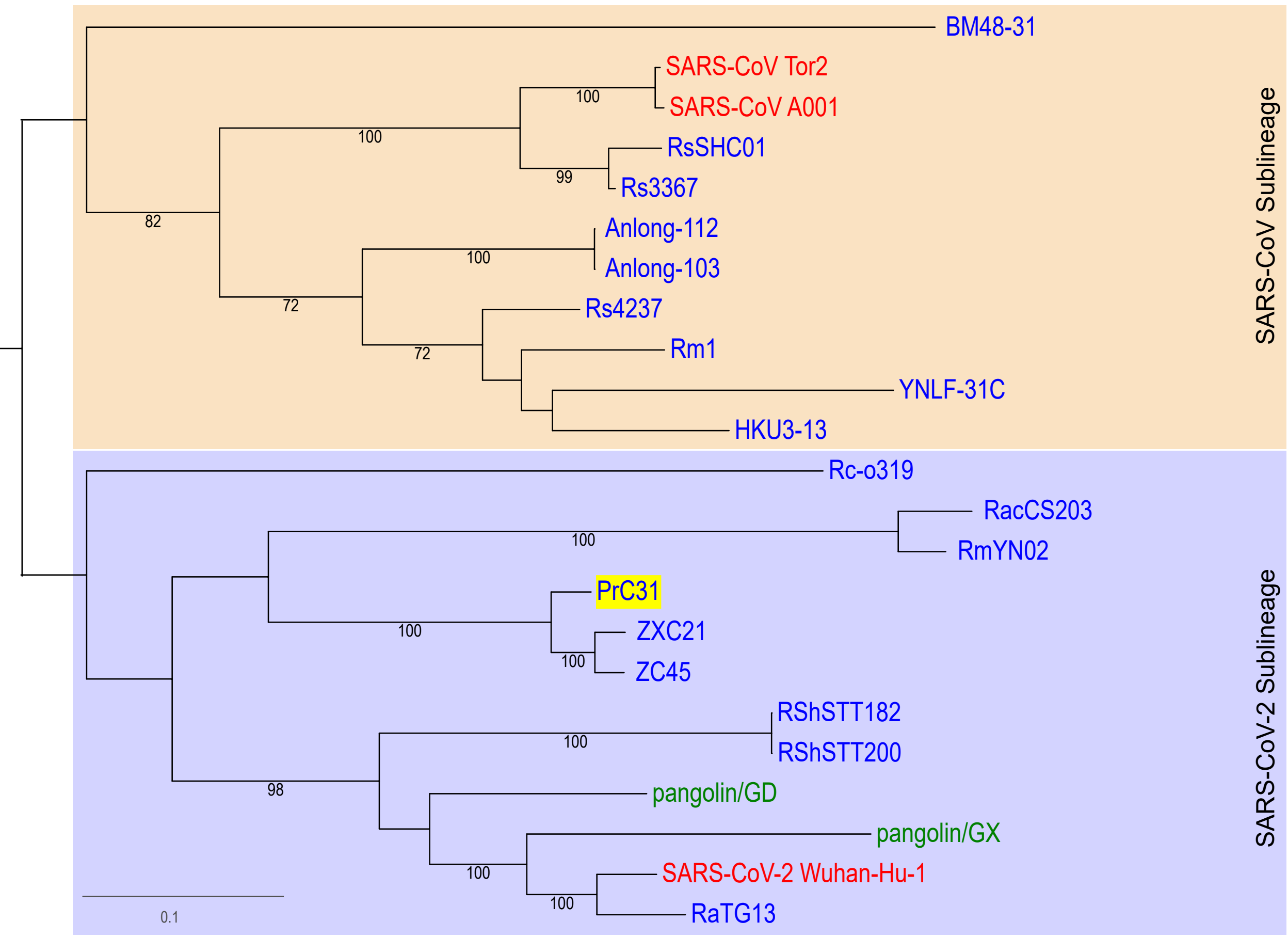

\section{B: RdRp gene}

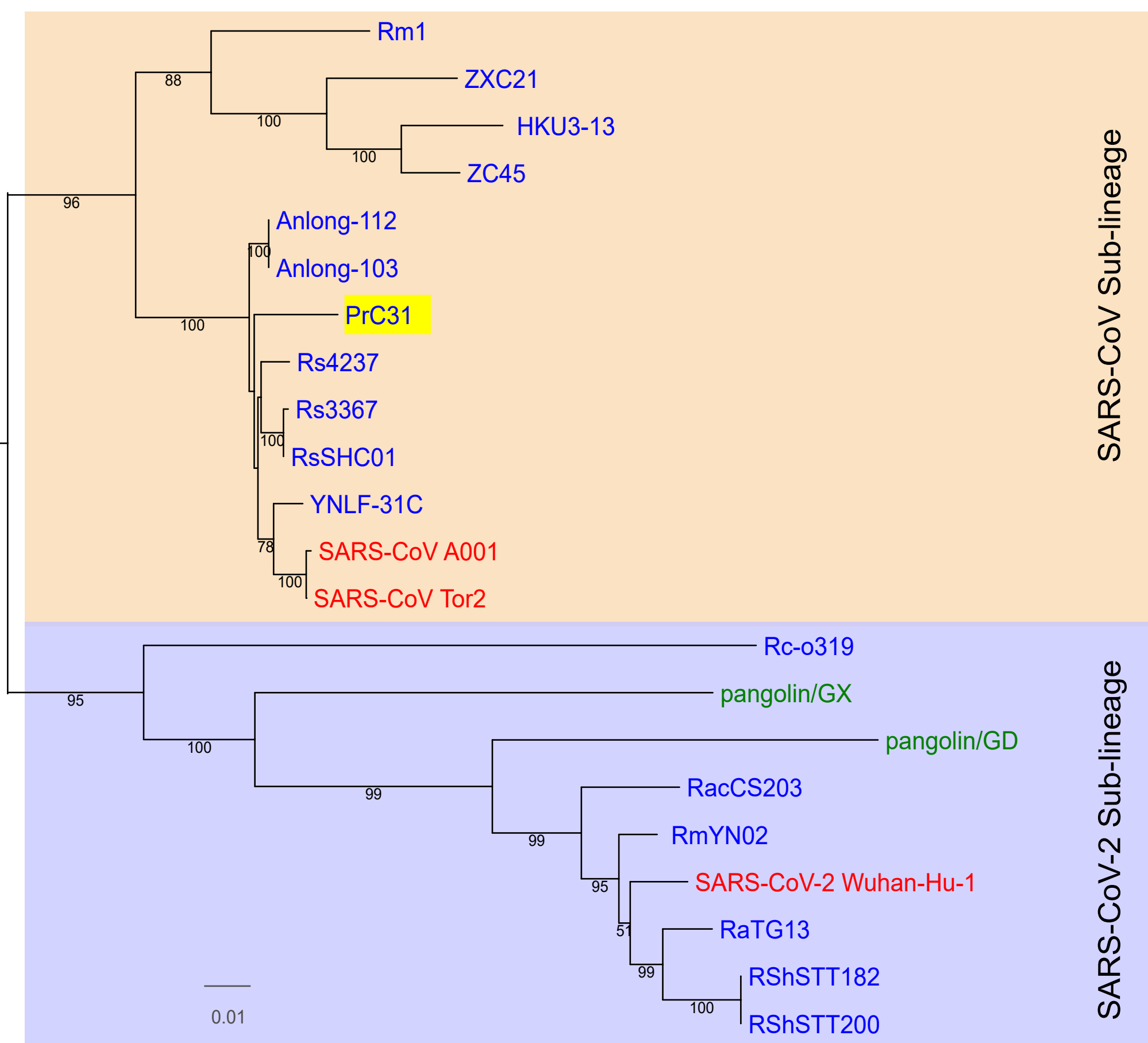

D: RBD

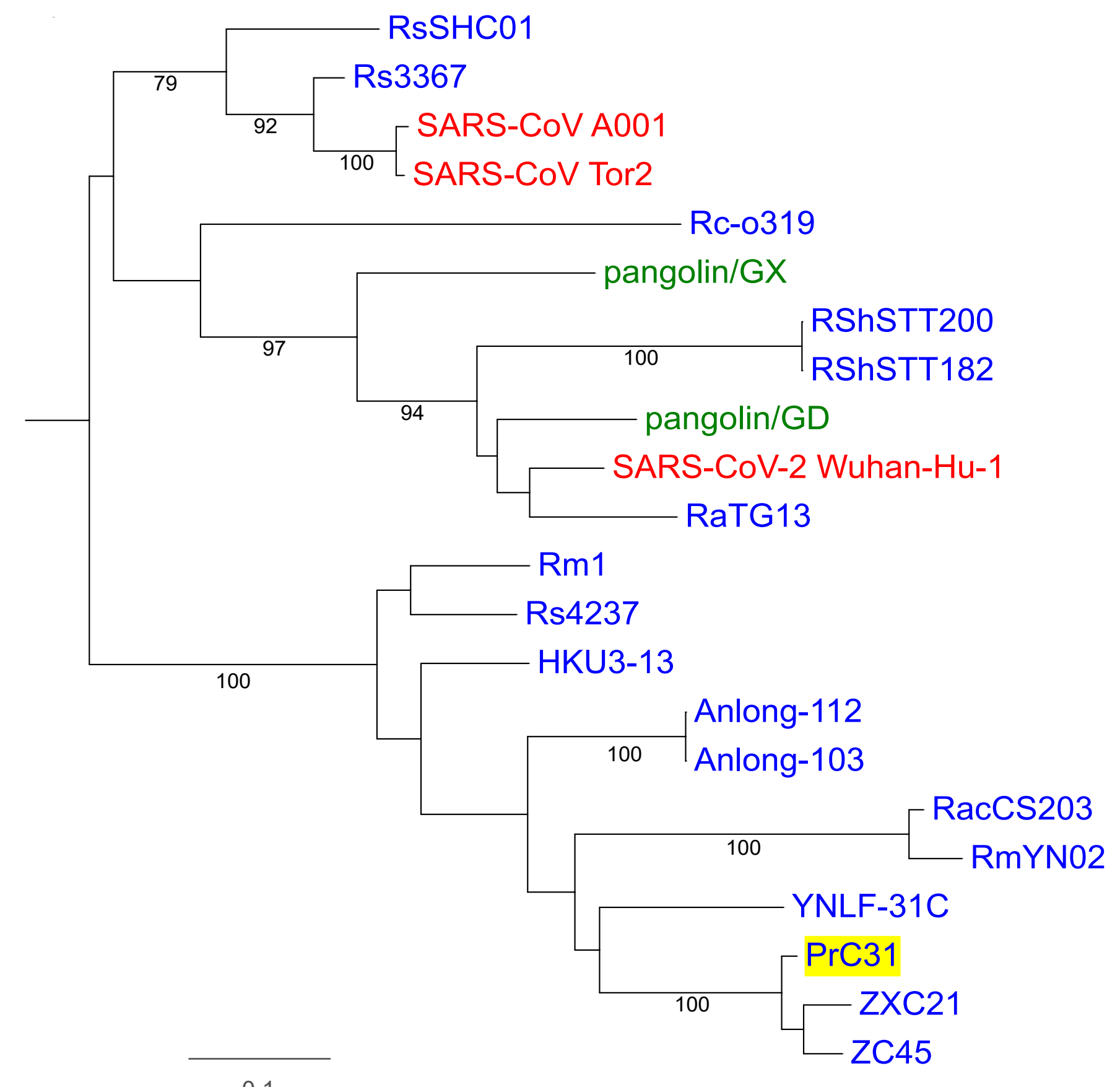




\section{Fig.3}
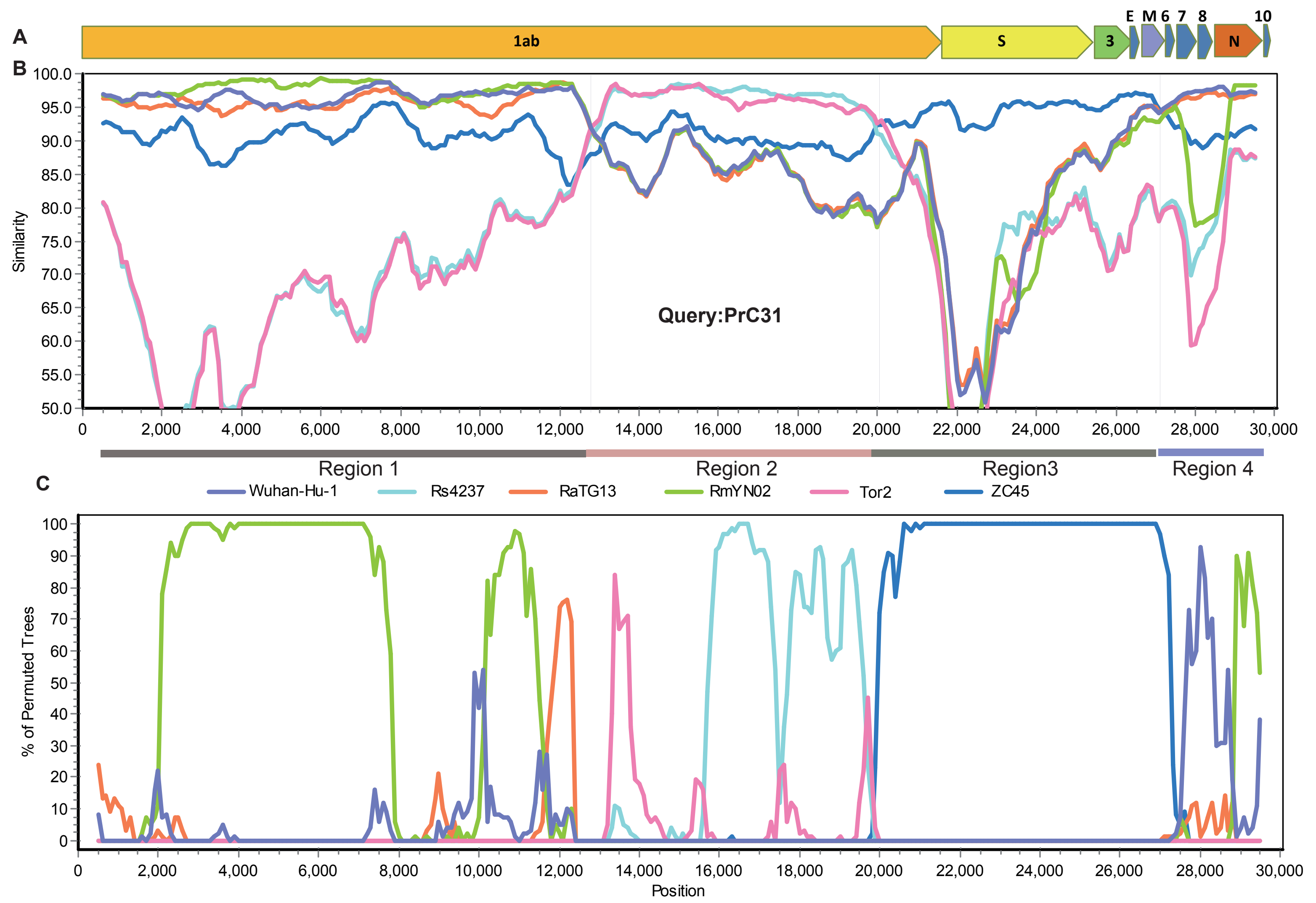


\section{A: Region 1}

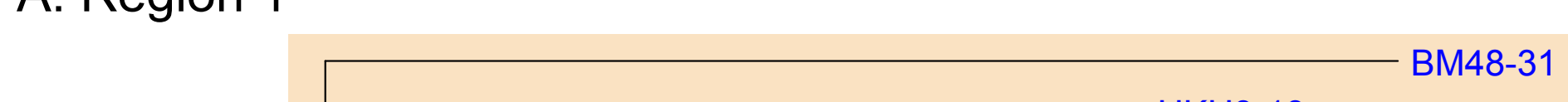

HKU3-13

1090Rxiv preprint doi: https://doi.org/10.1101/2021.03.17.4358\%3; 991 version posted March 19, 2083 The copyright holder for this preprint available under aCC-BY-NC-ND 4.0 Internation N NiEgnse $31 \mathrm{C}$
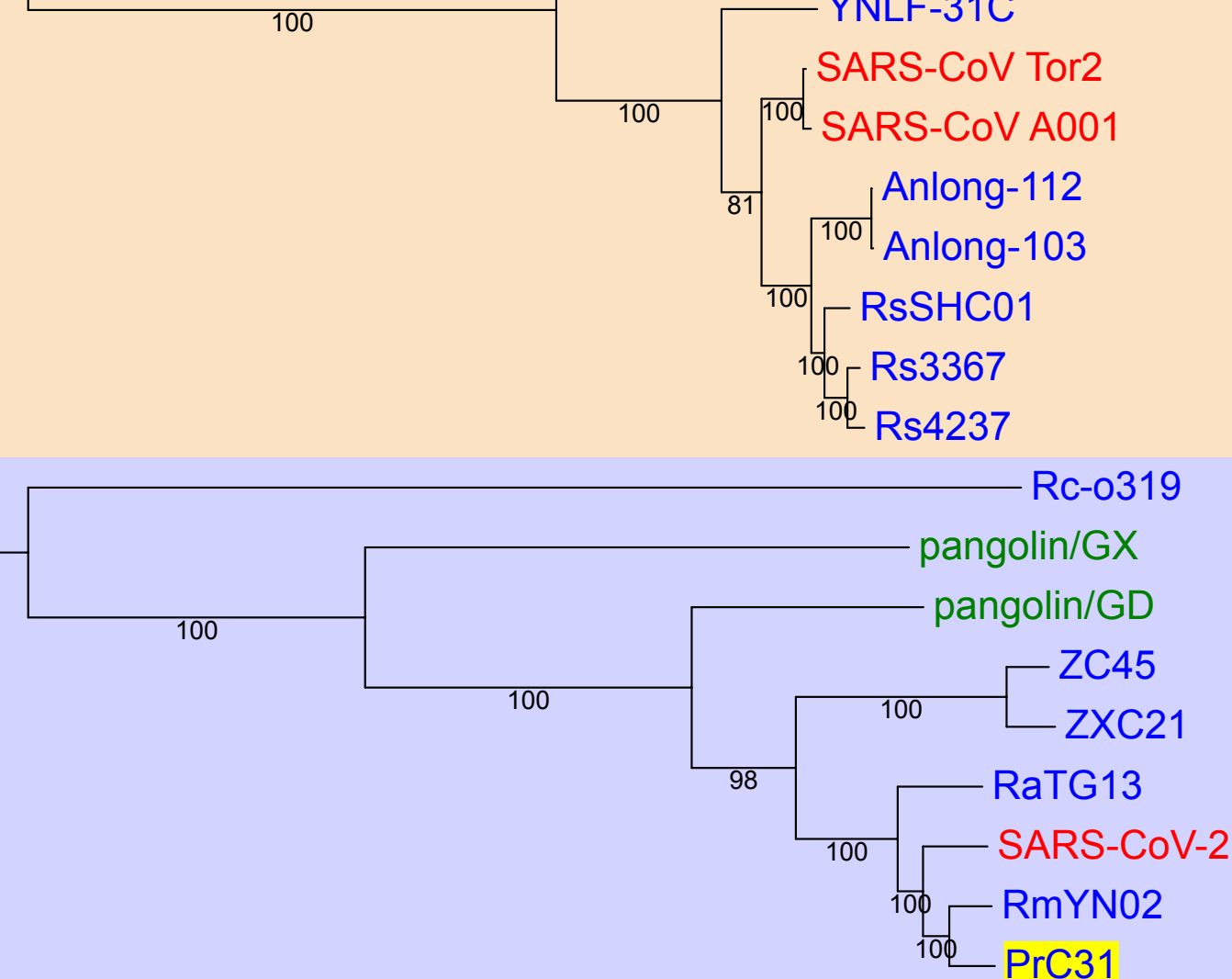

PrC31

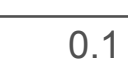

\section{C: Region 3}

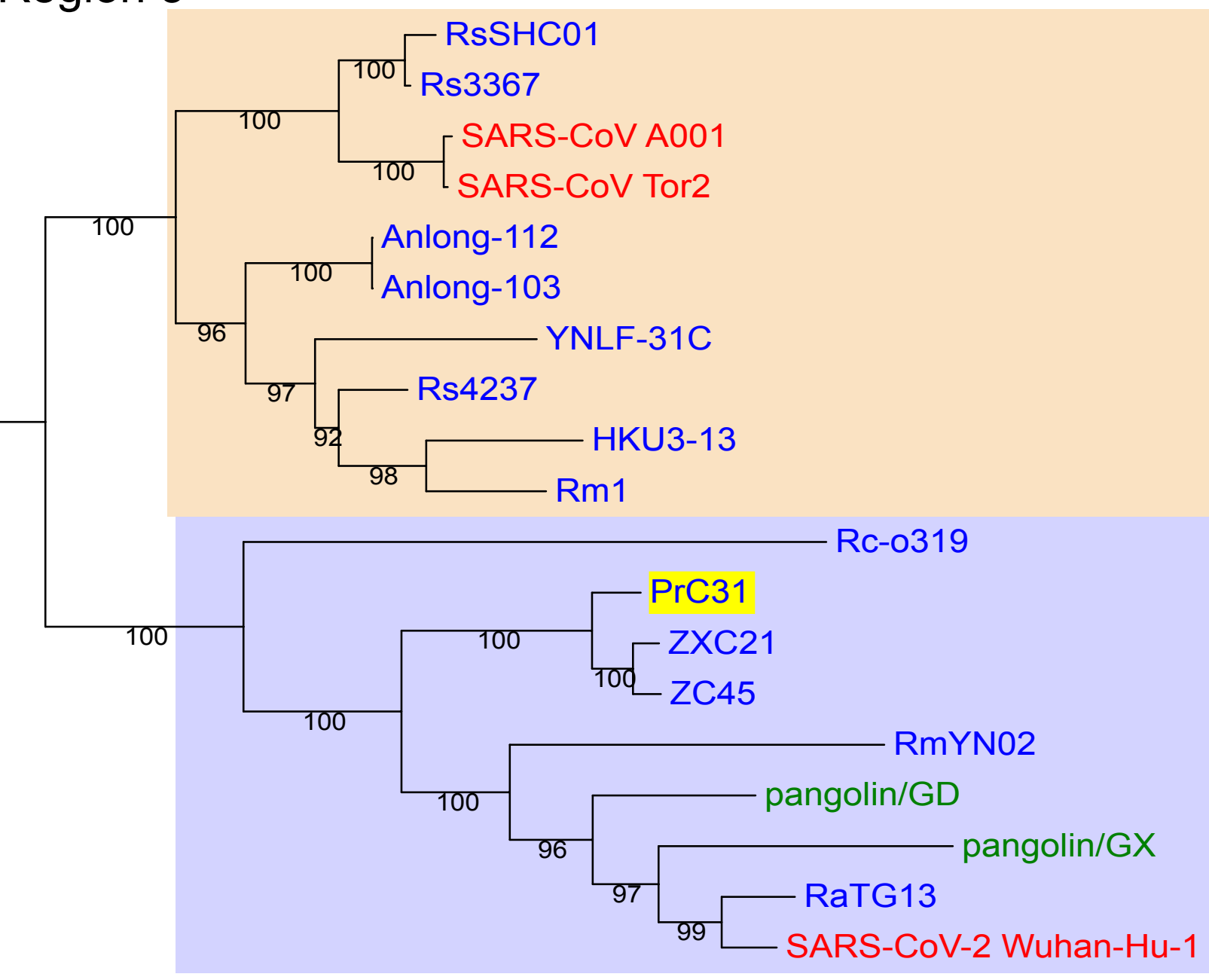

\section{B: Region 2}
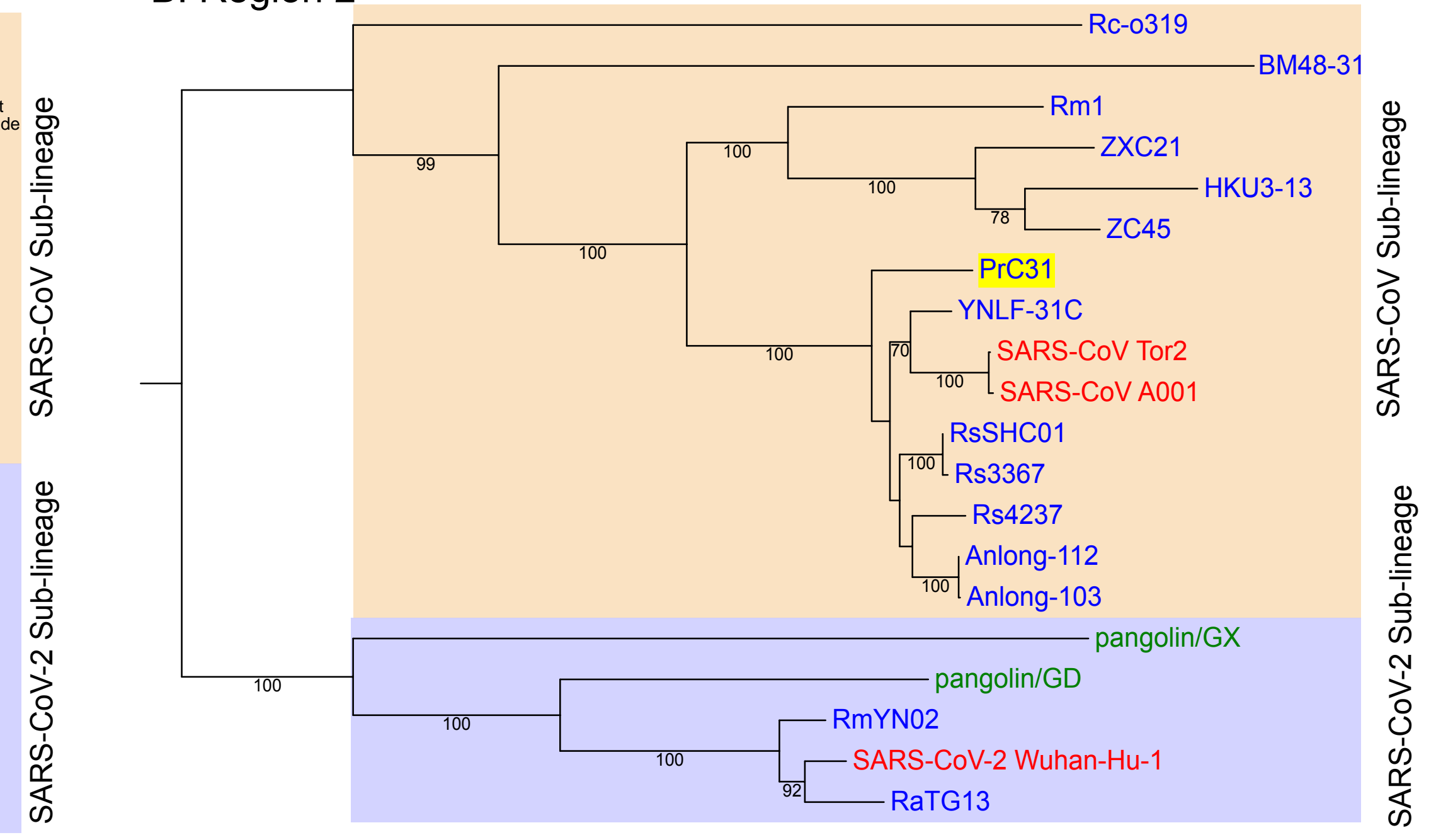

\section{D: Region 4}

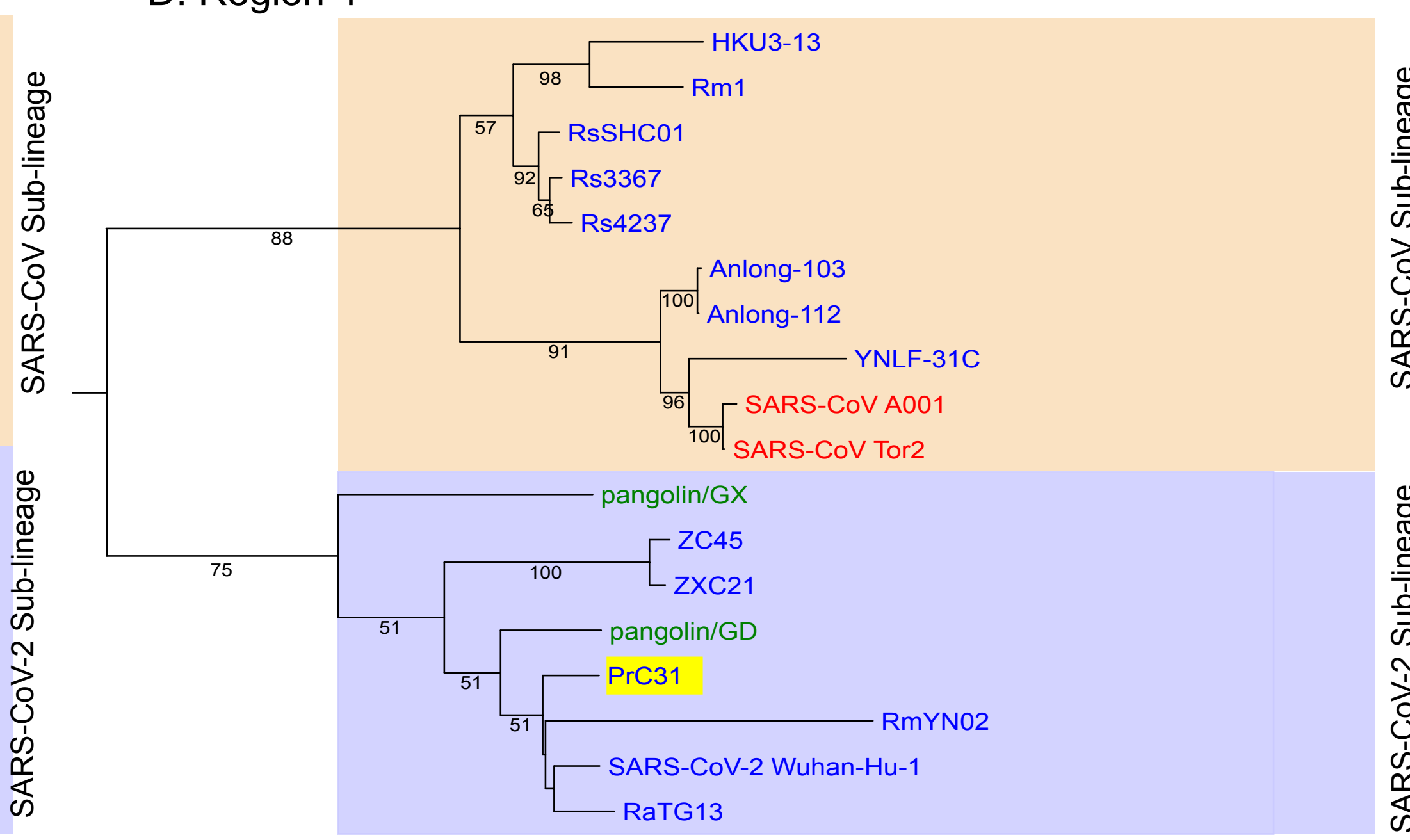

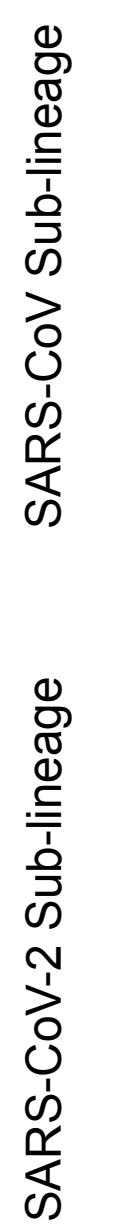

